\title{
Functional Endoscopic Tympanic Surgery Anatomical Bases
}

Alaa A. Abou-Bieh, MD1, Thomas J Haberkamp, MD²

${ }^{1}$ Department of Otolaryngology, Faculty

of Medicine, Mansoura University, Man-

soura, Egypt

${ }^{2}$ Cleveland Clinic, Cleveland, $\mathrm{OH}$, USA

\begin{abstract}
Objectives:

1- Describe the anatomical bases related to the concept of the functional endoscopic tympanic surgery (FETS) based on the fact that restoring adequate tympanic cavity ventilation is one of the main key factors to achieve this concept and restore normal middle ear functions.

2- Review and explain the possible theory and concept of the FETS in relation to these bases.
\end{abstract}

Methods: Fifty-five temporal bones were dissected, and the ventilation pathways were studied utilizing otoendoscopes of different angles and an operating microscope. In addition, the anatomy and relations of these pathways were studied in 200 temporal bones computed tomography scans (CT).

Results: The ventilation pathways of the tympanic cavity (with all of its subdivisions) and the mastoid air cells were variable and unique for each temporal bone. Basically, the ventilation patterns could be classified either directly from the eustachian tube or indirectly through communicating with other spaces via mucosal, ligamental and/or bony apertures or isthmi. Mesotympanum, protympanum, hypotympanum and anterior epitympanum type $\mathrm{C}$ were the only spaces directly ventilated, while retrotympanum with all of its recesses, anterior epitympanum types A and B, Prussak's space, von Troeltsch pouches, compartments of posterior epitympanum (medial and lateral superior and medial incudal spaces), antrum and mastoid air cells were indirectly ventilated. It was possible to approach most of the pathways endoscopically via transcanal or combined transcanal and transmastoid approaches.

Conclusions: Theoretically, ventilation pathways could be endoscopically approached and therefore restored in certain cases. CT scans are a possible tool to study them.

\section{Introduction}

Considering the evolution of the endoscopic middle ear surgery over the last four decades through hundreds of published researches, tens of books and many courses and conferences, the concept has passed from a diagnostic tool to a secondary complimentary tool or step to a complete surgical approach. ${ }^{1-6}$ However, until today the principle of incorporating endoscopes in the middle ear surgery was focusing mainly on the "approach" and most of efforts were to change the traditional microscopic surgery or combined microscopic and endoscopic surgery for disease eradication and / or hearing restoration into pure endoscopic approached surgery. Although, it is become well known, for over four hundred years since Bartholomeo Eustachi in 1576 has corrected the description of the Eustachian tube, that many of the middle ear diseases are result of ventilation problem and a lot of work have been done to describe treatment for the Eustachian tube dysfunction, the idea of developing a distinctive type of functional endoscopic middle ear surgery to treat the middle ear ventilation problems is not well established yet. ${ }^{7-9}$ The main principal of the functional endoscopic sinus surgery (FESS) is restoration of the 
normal sinus mucociliary clearance, drainage and ventilation functions through endoscopic removal of any even relatively minor anatomical or inflammatory pathological obstructive changes of the drainage pathways in the osteomeatal complex. ${ }^{10}$ Comparing to this principal, the principal of the functional middle ear (tympanic) surgery (FTS) or the functional endoscopic tympanic surgery (FETS), should be the same.

The aim of the current study was to describe the anatomical bases related to the concept of the functional endoscopic tympanic surgery (FETS) by reviewing the anatomy of the ventilation pathways and studying it in human temporal bones specimens. In addition, was to review and explain the possible theory and concept of the FETS in relation to these anatomical bases.

The proposed and most accepted theory for the tympanic cavity and mastoid ear cells ventilation tracks is by following the passage of the endothelial pouches of the primitive eustachian tube (sacci) to develop the middle ear air cell system.11 These pouches develop between the third and seventh fetal month when the gelatinous tissue of the middle-ear cleft is gradually absorbed and the primitive tympanic cavity develops by a growth, into the cleft, of an endothelial lined fluid pouch extending from the Eustachian tube (ET) and then four primary sacs or pouches bud out of it, they are saccus anticus, saccus medius, saccus superior and saccus posticus (Figure 1). ${ }^{12,13}$ Saccus medius, leaves the mesotympanum via the anterior tympanic isthmus (ITa) to extend posterior and forms the attic, and goes into the pars petrosal portion of the mastoid. Saccus superior, ascends over the styloid complex [pyramidal eminence $(\mathrm{Pe})$, styloid eminence (Se), and chordal eminence (CE)] and through the posterior tympanic isthmus (ITp) to form the antrum and squamosal portion of the mastoid together with the facial recess (FR) by descending behind the chordal ridge (CR) and droping downward on the lateral aspect of the facial canal (mFC). Saccus posticus, forms the hypotympanum, round window niche (RW), sinus tympani (ST), and inferior half of oval window niche (OW). Saccus anticus form the supratubal recess (STR). Prussak's space (Ps) develops from the medial saccule of the saccus medius. Where these endothelial sacs meet each other, the mucosal folds of the middle ear spaces are formed to make a complex system of individually ventilated spaces and tracks (Figure 2). ${ }^{12,14,15}$

\section{Anatomy of the Ventilation Pathways:}

The attic or epitympanum is almost completely separated from the mesotympanum by the ossicles and their folds except for two small but constant openings which it is proposed to call the isthmus tympani anticus (ITa) and the isthmus tympani posticus (ITp). The anterior opening lies posterior to the tensor tympani tendon (TTT) and anterior to the stapes (S) and long crus of the incus (ILP). The posterior opening is bounded posteriorly by the pyramidal process $(\mathrm{Pe})$ and posterior tympanic wall, laterally by the short process of the incus (ISP) and posterior incudal ligament (PIL) and anteriorly by the medial incudal fold (MIF) which extends from the short (ISP) to the long process (ILP) of the incus. Its medial boundary is the stapes (S) and the stapedial tendon $\left.{ }^{*}\right)$. The tensor fold (TF) is formed where the saccus anticus meets the anterior saccule of the saccus medius, while the cog (or the transverse crest) (C) is formed where the anterior saccule of the saccus medius meets the remaining saccus medius and forming the anterior epitympanic space $(A E)$ in one of its three variable types $(A, B$ and $C) .{ }^{16}$ The attic usually extends forward through the incisura tensoris (In) and anterior to the tensor tendon (TTT) as the anterior malleolar space (AMS) or anterior compartment of the attic. This space lies above the tensor fold (TF) which extends laterally from the semicanal for the tensor tympani muscle (TTC) to the anterior malleolar ligament (AML). The attic, however, may extend anteriorly only to the level of the tensor tendon (TTT) 
where it is limited by a medial extension of the superior malleolar fold (SMF) instead of by the tensor fold (TF) which in such an instance does not form. The space anteriorly would be in communication with the mesotympanum and eustachian tube. This space, when present, is called the supratubal space (STR). Posterior to the transversely-placed superior malleolar fold (SMF) lies the larger posterior compartment of the attic (PE). That portion of this compartment lateral to the superior incudal fold (SIF) may be considered as the superior incudal space (SIS) and that portion medial to the superior incudal fold as the medial incudal space (MIS). Laterally the floor of the superior incudal space is formed by the lateral malleolar fold (LMF) and by the lateral incudal fold(LIF) which extends posteriorly to the posterior incudal ligament (PIL). Medially the posterior compartment of the attic (PE) is separated from the mesotympanum by the dihedral-shaped medial incudal fold (MIF) which extends from both crura of the incus (ISP, ILP) to the pyramidal eminence $(\mathrm{Pe})$ and stapes $(\mathrm{S}) .{ }^{15,17,18}$

The space lies between the notch of Rivinus (NR), the anterior and posterior tympanic spines (A and PTyS) and the short process of the malleus (MSP). ${ }^{11}$ The medial and inferior aspects of Prussak's space (Ps) are formed, respectively, by the neck $(\mathrm{MN})$ and the short process of the malleus (MSP). ${ }^{11,12,16}$ It is limited above by the lateral malleolar fold (LMF) which arises from the junction of the malleus head ( $\mathrm{MHe}$ ) and neck (MN) and radiates out to insert on the entire bony rim of the notch of Rivinus (NR) and medial wall of the scutum (Sc), thus forming a firm roof for Prussak's space (Ps) and the floor of the lateral malleolar space (LMS). ${ }^{11,12}$ The space is limited anteriorly, and to some degree inferiorly, by the terminal flaring out of the annulus fibrosus (AF), the dense fibrocartilaginous ring to which the radial fibers of the drum attach, as it leaves the anterior tympanic spine (ATyS). As anteriorly when the annulus (AF) leaves the sulcus tympanicus (STy) it attach in part to the anterior tympanic spine (ATyS) then continues on as the stria membrana tympani anticus (SMTyA) to the short process (MSP), to radiate out to help form the floor of Prussak's space (Ps), to interdigitate with fibres of the lateral malleolar fold (LMF), and to attach to the bony rim of the notch of Rivinus (NR). ${ }^{11}$ Some authors describe the anterior aspect of the Prussak area (Ps) as being bounded by a very thin membranous fold among the tympanic membrane (TM) and the anterior malleolar ligament fold (AMF), which is inserted laterally onto the tympanic membrane (TM) and medially onto the neck of the malleus (MN) and the long process (MLP). Posteriorly, on leaving the posterior tympanic spine (PTyS), fibers of the annulus (AF) end at the malleus neck (MN), forming the posterior floor of the Prussak area (Ps).16,19,20 As posteriorly when the annulus (AF) leaves the sulucs tympanicus (STy) its outer fibres insert on the posterior tympanic spine (PTyS) or extend in the stria membrana tympani posticus (SMTyP) to the short process of the malleus (MSP) while its inner fibres insert on the medially-placed pretympanic spine (PreTyS) or radiate out, forming the supporting structure for the posterior malleolar fold (PMF) and attaching on the postero-medial aspect of the upper third of the malleus handle $(\mathrm{MH}){ }^{11} \mathrm{Be}$ tween the posterior malleolar fold (PMF) and the tympanic membrane (TM) lies the posterior pouch of von Troeltsch (VTP) which represents the Prussak's space (Ps) posterior wall. ${ }^{12,19}$ The lateral aspect is represented by the pars flaccid (PF) of the tympanic membrane (TM). ${ }^{19}$

Beneath the floor of the attic (AT) and in the upper mesotympanum there are three compartments: the inferior incudal space (IIS) and the anterior (VTA) and posterior (VTP) pouches of von Troeltsch. The inferior incudal space (IIS) extends from the inferior surface of the incus (I) laterally to the posterior malleolar fold (PMF). It is limited medially by the medial incudal fold (MIF) and anteriorly by the interossicular fold (IF) which lies be- 
tween the long crus of the incus (ILP) and the upper two thirds of the malleus handle $(\mathrm{MH})$. Between the posterior malleolar fold (PMF) and both the pars tensa (PT) and pars flaccid (PF) of the tympanic membrane (TM) lies the posterior pouch of von Troeltsch (vTP). The chorda tympani nerve (CTN) lies in the free margin of the posterior malleolar fold (PMF), although it may cross the posterior tympanum independent of this fold. The shallow anterior pouch of von Troeltsch (vTA) lies between that portion of the drumhead (TM) anterior to the malleus handle $(\mathrm{MH})$ and the anterior malleolar fold (AMF) which is draped on the anterior malleolar ligament (AML). 11,12,21,22

The posterior pouch of von Troeltsch (vTP) is the main route of ventilation of Prussak's space (Ps) and as this pouch opens at the most cranial portion of the mesotympanum, so in most subjects, ventilation of Prussak's space (Ps) occurs through the communication with the mesotympanum. Laterally and posterior in the attic (AT) the floor of the superior incudal space (SIS) is formed by the lateral malleolar fold (LMF) and by the lateral incudal fold (LIF) which extends posteriorly to the posterior incudal ligament (PIL). The entrance into Prussak's space (Ps) is usually located between the lateral malleolar fold (LMF) and the lateral incudal fold (LIF). ${ }^{11,12,19}$

Between the malleus handle $(\mathrm{MH})$ and the pars tensa (PT) and superior to the umbo (U) lies the shallow manubrial fold (MaF). Five folds may be recognized as stapedial folds, the obturatoria stapedis (ObS) between the crura (SC), the anterior stapedial fold (ASF) between promontory and anterior crus(aSC), the posterior stapedial fold (PSF) between promontory and posterior crus (pSC), the plica stapedis (Sp) between pyramidal eminence $(\mathrm{Pe})$ and the posterior crus (pSC) and the superior stapedial folds (SSF) which extend from the long crus of the incus (ILP) to either crus of the stapes (aSC, pSC) or from the facial canal to the crura (aSC, pSC). ${ }^{11,12}$

\section{Material and Methods}

The gross anatomy and anatomical variations of the tympanic cavity with all its subdivisions and the mastoid air cells system were studied in 55 Fresh frozen temporal bones (TBs) following all the proposed pathways for ventilation. To the best of our knowledge, all bones came from adult cadavers. There were 31 right temporal bones and 24 left. All bones were dissected by the same approach and all were studied utilizing an operating microscope and otoendoscopes with 00,300 and 700 angles and 2.7 and $3 \mathrm{~mm}$ diameters. In addition, the pathways anatomy and relations were studied in two hundred temporal bones CT scans (= 400 sides). These scans were randomly chosen regard less they were of normal individuals or showing any type of temporal bone pathology, but all were for adults.

\section{Results}

- The ventilation pathways of the tympanic cavity (with all of its subdivisions) and the mastoid air cells were variable and unique for each temporal bone.

- The ventilation patterns could be classified either (Figures 3 -10):

1- Direct ventilation routes from the eustachian tube: where it could be approached directly via the trans-tympanic endoscopic approach (through myringotomy or by elevation of the tympanomeatal flap) without the need to alter or remove any structure other than the mucosal folds, meatal wall bony buttresses and / or annular sulcus tympanicus. These included mesotympanum, protympanum, hypotympanum, anterior epitympanum type $\mathrm{C}$ and the inferior structures and spaces of the retrotympanum, including the sinus tympani, the inferior part of the oval window and the round window.

2- Indirect ventilation routes through communicating with other spaces via mucosal, ligamental and/or bony apertures or one of the tympanic or epitympanic isthmi: where it 
could be approached indirectly only via the trans-tympanic endoscopic approach (through elevation of the tympanomeatal flap) after complete or partial removal of any of the ossicles, mucosal folds, ligaments, muscle tendons, chorda tympani nerve, parts of the tympanic membrane or annulus, meatal wall bony buttresses and / or annular sulcus tympanicus. Or, it needed trans-mastoid or combined trans-canal and trans-mastoid ap- proaches to approach them. These included anterior epitympanum types A and B, Prussak's space, von Troeltsch pouches, compartments of posterior epitympanum (medial and lateral superior and medial incudal spaces), antrum and mastoid air cells and the superior structures and spaces of the retrotympanum, including the facial recess (full depth) and the superior part of the oval window.
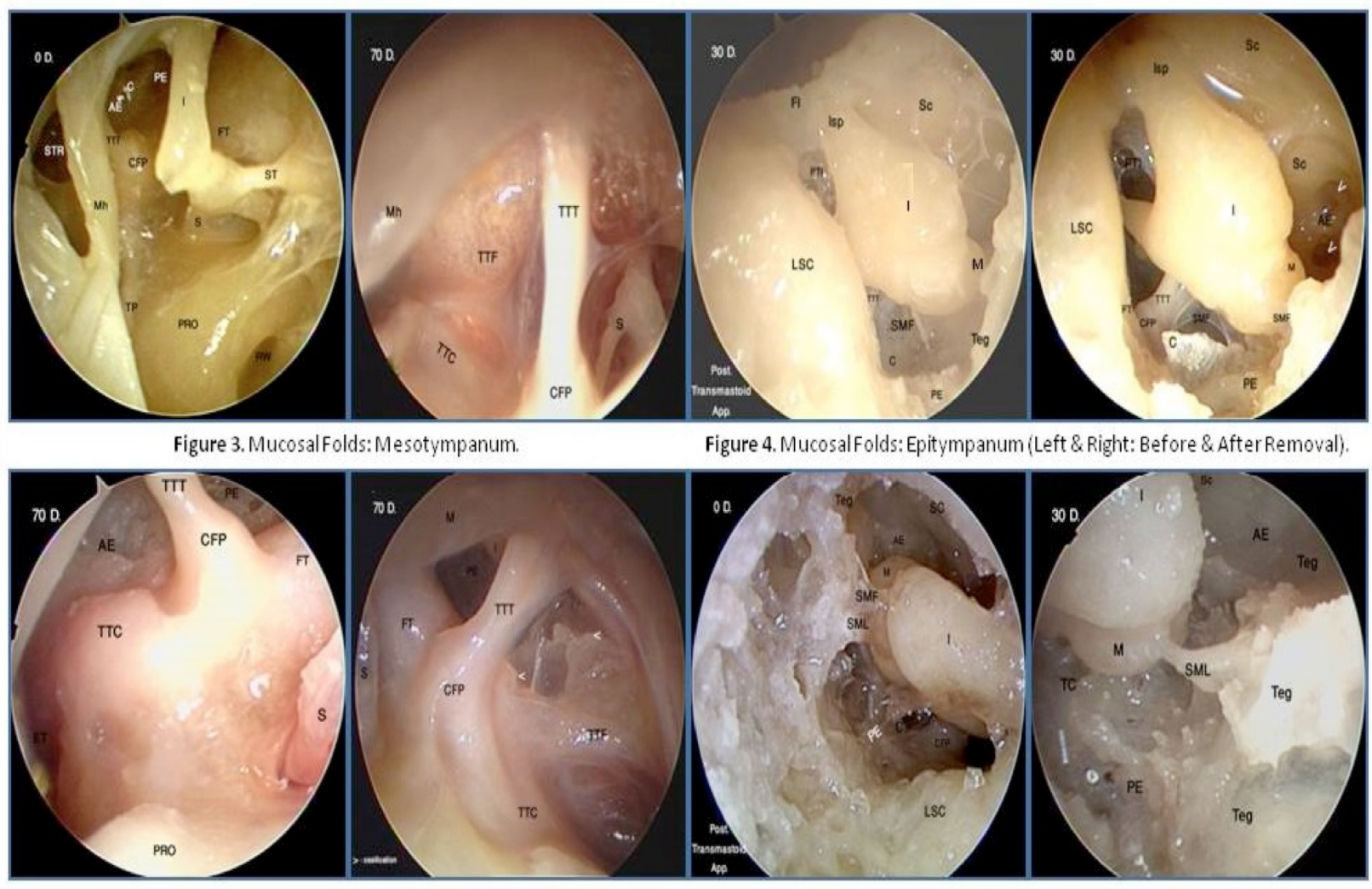

Figure 5. Anterior Epitympanum: Types A, B \& C From Right to Left.

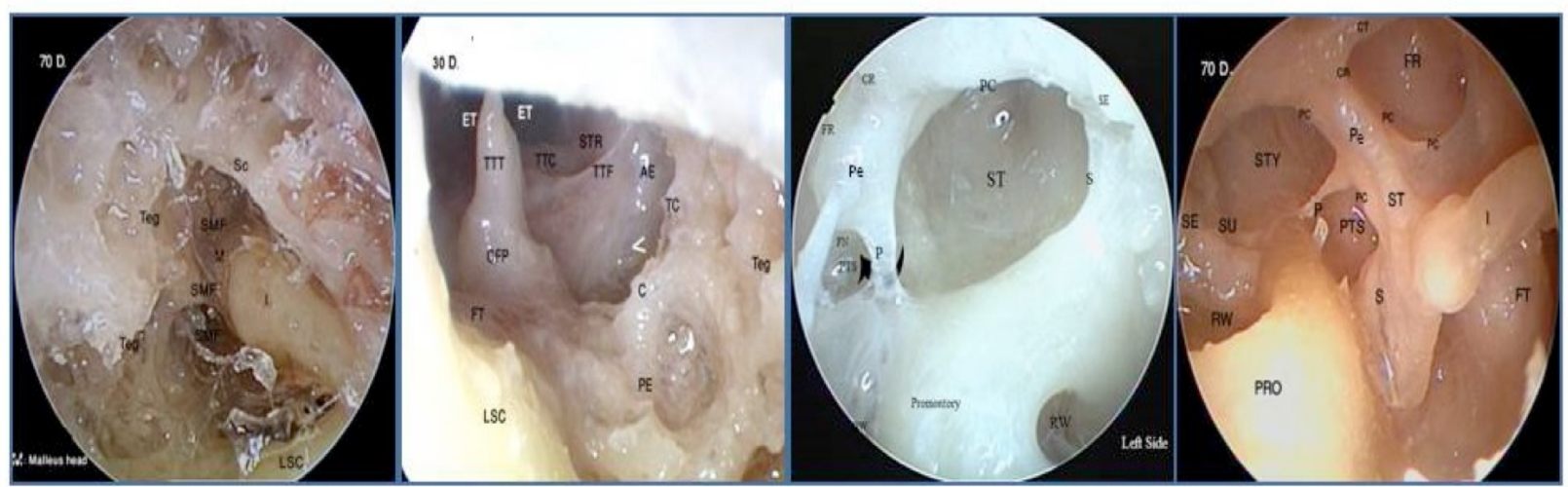

Figure 6. Posterior Epitympanum. (Lateral \& Medial from Left to Right)
Figure 7. Retrotympanum. (Inferior \& Superior from Left to Right) 
Flaxseed and corn oils on Myocardial Infarction Alaa A. et al. 24

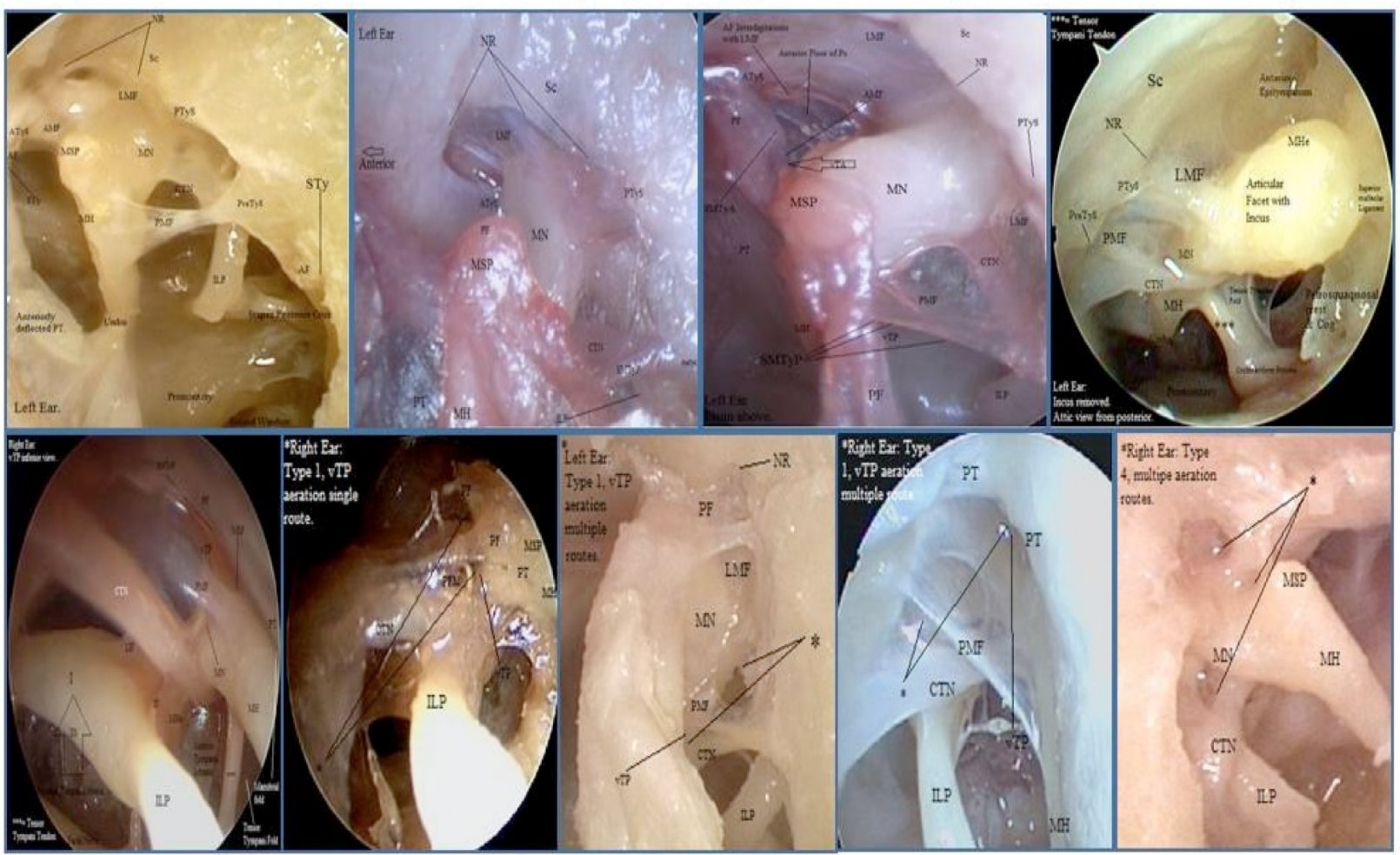

Figure 8. Prussak's Space

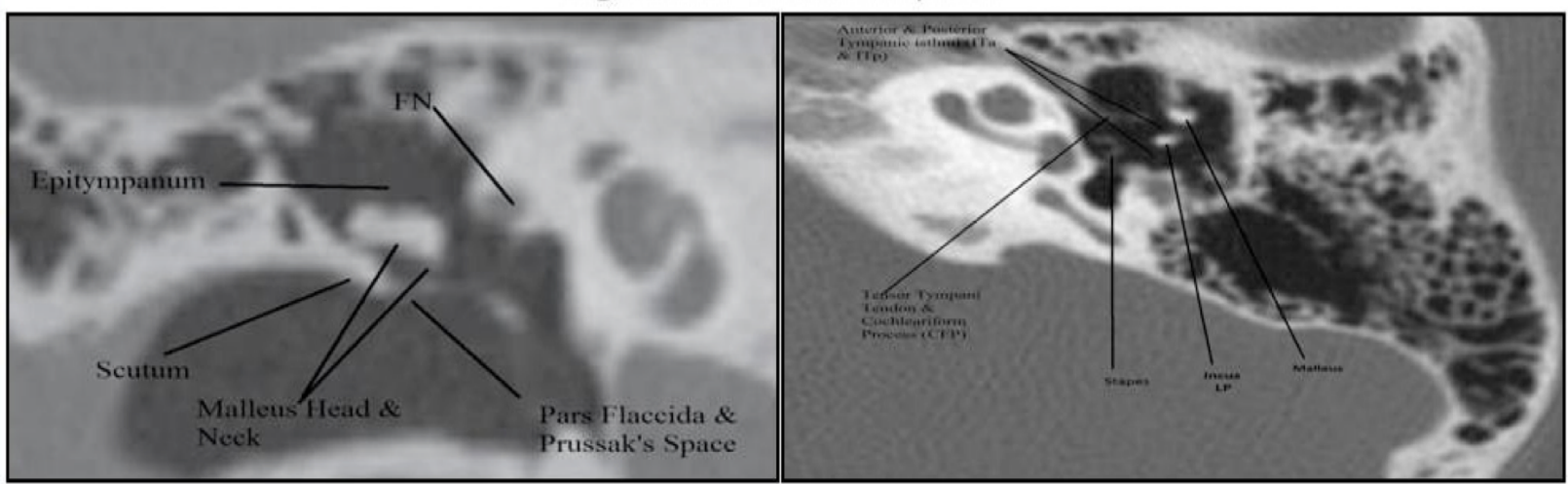

Figure 9. Coronal CT Prussak's Space

Figure 10. Axial CT Level of Tympanic Ismthi 


\section{Discussion}

The current cadaveric anatomical study has shown that the ventilation pathways could be endoscopically approached either directly or indirectly and that CT scans are a possible tool to study them. Although many studies has reviewed approaching different tympanic areas, 19 the idea of investigating, tracking and scanning the tympanic ventilation pathways was not discussed in literature before and no published work proposed the concept of the functional tympanic endoscopic surgery.

The current study results have demonstrated that theoretically the concept of the FTS and FETS can be applied to the tympanic cavity ventilation pathways to restore them in certain cases. However, this should be studied in detail first in real life cases. In addition, some issues should be addressed in the following studies, like the difference in the ventilation pathways between adults and pediatrics and the difference between ventilation disorders due to distal Eustachian tube obstruction or dysfunction and proximal Eustachian tube or tympanic obstruction. Another issue which should be addressed is the role of mucosal folds in ventilation, because either traditional or endoscopic approaches destroy them significantly. However, the most important issue that should be addressed and proved with the advance in research for the concept of the FTS would be "whether the current undisputed ventilation theory is accurate or not". Another issue that was highlighted by the results of the current study is the true value and accuracy of the currently used methods of ventilation, starting from simple myringotomy, passing by all kinds of ventilation, drainage and pressure equalizing tubes and ending by the role of different types of mastoidectomies +1 meatoplasties in ventilation.

\section{Conclusions}

Theoretically, based on the ventilation pathways hypothesis, these pathways anatomical locations and distribution could be endoscopically approached and therefore restored in certain cases. CT scans are a possible tool to study their anatomy.

\section{References}

1- Mer SB, Derbyshire AJ, Brushenko A, Pontarelli DA. Fiberoptic endotoscopes for examining the middle ear. Arch Otolaryngol. 1967;85:387393.

2- Eichner H. A new optical system in tympanic membrane and middle ear endoscopy. Endoscopy. 1981;13(5):211-313.

3- Thomassin JM, Korchia D, Doris JM. Endoscopic-guided otosurgery in the prevention of residual cholesteatomas. Laryngoscope. 1993;103:939-943.

4- Bader-el-Dine M. Value of ear endoscopy in cholesteatoma surgery. Otol Neurotol. 2002;23:631-635.

5- Rehl RM, Oliaei S, Ziai K, Mahboubi H, Djalilian $\mathrm{H}$. Tympanomastoidectomy with otoendoscopy. Ear Nose Throat J. 2012;91:527-532.

6- Tarabichi M1, Ayache S, Nogueira JF, Al Qahtani M, Pothier DD. Endoscopic management of chronic otitis media and tympanoplasty. Otolaryngol Clin North Am. 2013;46(2):155163.

7- Choulant, L. History and bibliography of anatomic illustration. Trans. and annotated by Mortimer Frank. New York: Hafner; 1962. p. 200-204.

8- Poe DS, Hanna BM. Balloon dilation of the cartilaginous portion of the eustachian tube: initial safety and feasibility analysis in a cadav- 
er model. Am J Otolaryngol. 2011;32(2):115123.

9- Tarabichi M, Najmi M. Site of eustachian tube obstruction in chronic ear disease. Laryngoscope. 2015;125(11):2572-2575.

10- Kennedy DW, Zinreich SJ, Rosenbaum AE, Johns ME. Functional endoscopic sinus surgery. Theory and diagnostic evaluation. Arch Otolaryngol. 1985;111(9):576-582.

11- Proctor B. Surgical Anatomy of the Ear and Temporal Bone. New York: Thieme Medical Publishers; 1989. p. 159-196.

12- Proctor B. The development of the middle ear spaces and their surgical significance. J Laryngol Otol. 1964;78:631-648.

13- Hammar JA. Studien iiber die Entwieklung des Vorderdarms und einiger angrenzender Organe. Arch, fiir mikr. Anat. 1902;Vol. 59.

14- Chatellier HP, Lemoine J. Le diaphragme interatticotympanique du nouveau-ne. Ann Otolaryngol Chir Cervicofac. 1946;13:534566.

15- Palva T, Ramsay $H$. Incudal folds and epitympanic aeration. Am J Otol. 1996;17:700-708.
16- Palva T, Johnsson L. Epitympanic compartment surgical considerations: reevaluation. Am J Otol. 1995;16:505-513.

17- Proctor B. Epitympanic mucosal folds. Arch Otolaryngol. 1971;94(6):578.

18- Palva T, Ramsay H, Northrop C. Color Atlas of the Anatomy and Pathology of the Epitympanum. 1st ed. Basel: Karger; 2001. p. 3-49.

19- Marchioni D, Alicandri-Ciufelli M, Grammatica A, et al. Lateral endoscopic approach to epitympanic diaphragm and Prussak's space: a dissection study. Surg Radiol Anat. 2010;32:843-852.

20- Palva T, Ramsey H. Aeration of Prussak's space is independent of the supradiaphragmatic epitympanic compartment. Otol Neurotol. 2007;28:264-268.

21- Donaldson JA, Duckert LG, Lambert PM, Rubel EW. Anson - Donaldson Surgical Anatomy of the Temporal Bone. New York: Raven Press, 1992. p. 40-98.

22- Gulya AJ. Gulya and Schuknecht's Anatomy of the Temporal Bone with Surgical Implications. 3rd ed. New York: Informa; 2007. p.8092. 